\title{
Unfixed Resources: Perceived Costs, Consumption, and the Accessible Account Effect
}

\author{
CAREY K. MOREWEDGE \\ LEIF HOLTZMAN \\ NICHOLAS EPLEY*
}

Consumption depletes one's available resources, but consumers may be unaware of the total resources available for consumption and, therefore, be influenced by the temporary accessibility of resource accounts. Consistent with this possibility, consumers in four experiments perceived a unit of consumption to be smaller and consequently consumed more, when large resource accounts of money, calories, or time (e.g., the money in their savings account) were made temporarily accessible compared with when small resource accounts were made temporarily accessible (e.g., the money in their wallet). Manipulating the cognitive accessibility of resources available for consumption influences both subjective judgment and behavior.

C onsumption of any commodity - from money to calories to time-by definition involves the depletion of resources from some larger pool or account. A \$15 compact disc depletes \$15 from one's overall wealth. A 240-calorie candy bar depletes 240 calories from one's ideal caloric intake. And a 2-hour home project depletes 2 hours from one's 24-hour day. Consumption, in other words, is both an absolute and relative enterprise. Every expense depletes one's available resources by an absolute amount, but the more resources one has available for consumption, the

\footnotetext{
*Carey $\mathrm{K}$. Morewedge is assistant professor of social and decision sciences at Carnegie Mellon University, 208 Porter Hall, Pittsburgh, PA 15213 (morewedge@post.harvard.edu). Leif Holtzman is an analyst at Digitas, 25 Bay State Road, Boston, MA 02215 (holtzman@post.harvard.edu). Nicholas Epley is an assistant professor of behavioral science at the University of Chicago, Graduate School of Business, 5807 South Woodlawn Avenue, Chicago, IL 60637 (epley@chicagogsb.edu). This research was supported by a dissertation grant from the Institute for Quantitative Social Science at Harvard University and a John Parker Scholarship from the Graduate Society of Fellows of Harvard University awarded to Morewedge, and grants from the National Science Foundation (SES 0241544) and the James S. Kemper Foundation Faculty Research Fund awarded to Epley. The authors thank Broadway Market and the Harvard Coop in Cambridge, Massachusetts, for the generous use of their facilities and cooperation; David Held, Kathleen Lee, Rebecca Levine, Evan Rapien, Laura Saslow, Aram Seo, Lisa Shu, Hillary Steinbrook, Bree Tsee, Alicia Warlick, and Erin Whitchurch for their help conducting these experiments; and John Deighton, the associate editor, and several reviewers for their suggestions.
}

John Deighton served as editor and Stephen Nowlis served as associate editor for this article.

Electronically published June 1, 2007 weaker the proportional impact of any one unit of consumption on one's overall resources. The extent to which consumption depletes one's resources can therefore be modeled as a very simple fraction, with the absolute cost of a good in the numerator- "How much does it cost?"- and the resources available for consumption in the denominator- "How much do I have to spend?" Whether a person can afford a particular unit of consumption depends not just on the price of consumption but on whether one can afford the purchase as well. The cost of a good is often very well known or intuitively easy to calculate, but the resources available for consumption must often be calculated or inferred. In this article we suggest that people do not fully consider the total amount of resources available for their consumption, and consumer evaluations and decisions are therefore highly sensitive to the size of the resource account that just happens to be cognitively accessible at the time of judgment. In particular, we predict that large accessible resource accounts make the objective cost of an item seem subjectively less expensive by comparison, thereby increasing the likelihood of consumption, whereas small accessible resource accounts make the objective cost of an item seem subjectively more expensive, thereby decreasing the likelihood of consumption.

Economic models of human decision making generally assume that consumers are aware not only of the numerator in the consumption equation (the absolute cost of a good) but the denominator as well (the amount of overall financial resources available for consumption), and therefore they use both kinds of information when making purchase decisions. 
For example, the dominant economic theory of financial savings behavior - the life-cycle hypothesis (Ando and Modigliani 1963) - assumes that people seek a relatively constant level of consumption across their lifetimes and that they therefore consume relatively less of their available income during periods of unusually high income and spend relatively more during periods of unusually low income. Consumers are assumed to calculate, at least implicitly, not only the cost of goods and the amount of money currently at their disposal (including all assets) but also the amount of money and assets they will earn during their lifetimes.

Existing psychological research has focused much attention on the numerator in this simple consumption equation"How much does it cost?"-investigating how altering the description of objective costs associated with consumption can influence consumption decisions. People are more likely to donate to charities, for instance, when the cost of donation is described as the cost incurred per day rather than as the cost incurred per month (Gourville 1998). This "pennies-aday" result influences consumption-in this case financial donations - not by altering the perceived resources people have available for consumption but, rather, by altering the perceived cost of a commodity or purchase (Chandran and Menon 2004). People are also more likely to purchase a good when its objective cost compares favorably with other similar goods (when a good is less expensive than others) than when it compares unfavorably with other similar goods (when a good is more expensive than others; Herr 1989), are more likely to indicate that they would buy a good on sale as a function of the objective amount of money saved compared with the overall cost of the good (Kahneman and Miller 1986; Kahneman and Tversky 1979), and are more likely to purchase an individual good when it is thought of in relation to the total expense of a group of goods of which it is a part (Leclerc, Schmitt, and Dubé 1995). Although consumers who wish to maximize the expenditure of their resources should always compare an expense to alternative ways to spend their resources, consumers do not engage in such extensive comparisons (Hsee et al. 1999; Kahneman and Miller 1986) and are thus highly sensitive to contextual information that may induce perceptual contrasts when making evaluations (Helson 1964; Parducci 1995).

We focus our research, however, on the denominator of the simple consumption equation- "How much do I have to spend?"- to obtain a more complete picture of consumption decisions. Obtaining an estimate of the total amount of resources available for consumption is not impossible, but it appears that such estimates are not spontaneously generated at the time of judgment (Heath and Soll 1996; Soman and Cheema 2002; Thaler 1985). Just as consumption decisions are influenced by comparisons between commodities and the goods to which they are compared, purchase decisions may be similarly influenced by comparisons between commodities and particular resource accounts that consumption would deplete. Although a person may recognize that a $\$ 15$ compact disk would deplete an equal absolute amount from the amount of money in one's wallet, money in one's checking account, or in one's estimated net worth, that person may be most willing to purchase the compact disk when its cost is compared with a large resource account. And as the amount of resources available for consumption is spontaneously generated at the time of judgment, consumption decisions may thus be determined by the account of resources that happens to be considered at the time of judgment. Large resource accounts - such as the total amount of money in one's bank account or the number of days in a year-are likely to make any individual unit of consumption appear subjectively smaller by comparison and therefore increase the likelihood of consumption compared with small resource accountssuch as the total amount of money in one's wallet or the number of days in a week.

Indeed, research on consumption across different transaction mediums demonstrates that people spend more for the same items when paying with credit cards than when paying with cash. These results are attributed, at least in part, to the decoupling, with a credit card, of a purchase and its drain on one's financial resources, thereby making the subjective costs of credit purchases appear to be less "painful" than cash purchases (Gourville and Soman 1998; Soman 2001). This is not surprising in normal consumer contexts given liquidity constraints (Feinberg 1986; Hirschman 1979), but people in auctions with no liquidity constraints also bid up to twice as much for the same good when bidding with a credit card than when bidding with cash (Prelec and Simester 2001). These findings suggest that the subjective cost of a commodity influences not only whether or not its consumption appears justifiable but also the decision to consume a commodity as well.

Because people are unlikely to consider fully the resources available for consumption, we suggest in this article that consumption decisions are also determined by the size of the resource account cognitively accessible at the time of judgment. More specifically, we predict that people are more likely to consume a given commodity when a large account of their resources is readily accessible than when a small account of their resources is accessible. The accessibility of resource accounts may moderate the subjective costs of consumption through a simple comparison process, making objects appear relatively more or less expensive in comparison to the total amount of resources in that account, without altering consumers' perceptions of the objective cost of a good. For instance, people thinking of the money available in their checking account can recognize that a compact disk costs $\$ 15$ but can also perceive its cost to be relatively smaller in a subjective sense than people thinking of the money available in their wallet. In other words, the smaller the resulting fraction of the absolute cost of an expense over the resource account that is thought of at the time of judgment, the smaller the subjective cost of that expense's consumption, and the more likely one is to consume it.

This "accessible account effect" makes three specific predictions that we test in four experiments. First, experimentally manipulating the accessibility of small versus large 
resource accounts should influence consumption, as costs should appear smaller in comparison to large accounts of one's resources than in comparison to smaller accounts. In other words, people will be more likely to expend their resources when a particular expenditure would deplete a relatively small, as opposed to a relatively large, proportion of the resource account that is cognitively accessible.

H1: People will consume a larger absolute amount of resources (e.g., money, calories, or time) when large resource accounts are cognitively accessible than when small resource accounts are cognitively accessible.

Second, the size of the cognitively accessible resource account will not influence judgments of the objective cost of an expense but will, rather, influence its subjective costs - how expensive or affordable a particular instance of consumption is perceived to be-and the "psychological pain" associated with that expenditure (Prelec and Loewenstein 1998). Goods to be consumed should therefore appear subjectively less costly or expensive when consumers consider how they would deplete a large resource account rather than a smaller resource account, without changing the objective cost associated with the commodity.

H2: The subjective cost of consumption will be more strongly influenced by the accessibility of one's resources - to be smaller when large accounts are cognitively accessible and larger when small accounts are cognitively accessible - than the objective or absolute cost of the expense.

Third, the subjective cost of a commodity will mediate the impact of accessible resource accounts on its consumption. People should be more likely to consume a commodity when large accounts are cognitively accessible because the commodity appears, subjectively, to be less expensive than when small resource accounts are cognitively accessible.

H3: The subjective cost of consumption will mediate the impact of accessible resource accounts on actual consumption.

We tested these hypotheses across a series of experiments measuring consumption of three major commodities-money, calories, and time. Experiments 1 and 2 investigated whether manipulating the accessibility of large versus small resource accounts can influence the consumption of money and calories (hypothesis 1). Experiments 3 and 4 investigated whether manipulating the accessibility of large versus small resource accounts can also influence the subjective cost of a good (hypothesis 2), and whether the impact of accessible resource accounts on the consumption of commodities is mediated by the subjective cost of the good (hypothesis 3).

\section{EXPERIMENT 1: FINANCIAL CONSUMPTION}

Experiment 1 utilized a manipulation of perceived account size that simply made a small or large account cognitively accessible without explicitly leading participants to indicate a numerical value associated with that resource account, and then the experiment measured how much participants purchased at a local market. Before shopping, participants in this experiment simply identified features of their small and large financial accounts by indicating what objects were contained in their wallet or what kinds of financial accounts they possessed (without indicating the objective value of any of these objects). We predicted that participants randomly assigned to think about small resource accounts would purchase less than participants randomly assigned to think about large resource accounts (hypothesis 1).

\section{Method}

Participants. One hundred and fifteen customers (43 males and 72 females, $M_{\text {age }}=43.8, \mathrm{SD}=12.9$ ) of Broadway Market in Cambridge, Massachusetts, participated in exchange for a gourmet chocolate bar. Broadway Market sells packaged foods, fruits and vegetables, meats, and deli sandwiches, among other miscellaneous grocery items. Data from three participants who spent more than three standard deviations from the overall mean were removed from the analyses, leaving data from a total of 112 participants.

Procedure. Before entering the market, customers completed a questionnaire on "incidental memory." Participants randomly assigned to the small account condition were instructed to indicate whether or not five items were currently in their wallet or purse: pictures, library cards, credit cards, photo identification cards, and cash. Participants randomly assigned to the large account condition indicated whether or not they currently possessed five kinds of financial accounts: checking, savings, bonds, stocks, and certificates of deposit. Next, customers indicated the total number of each type of item or account that they possessed (e.g., three library cards or three checking accounts). In other words, participants were asked to indicate the number of items or accounts they possessed, not the objective value of these items or accounts. Next, participants wrote their birth date on the questionnaire so that it could later be matched up with their shopping receipt, and then they began shopping.

When they were finished shopping, customers showed their receipt to an experimenter who recorded the pretax subtotal and transaction medium (i.e., credit card, checking or debit card, or cash). Customers then reported their birth date (so their receipt could later be matched with their questionnaire), were debriefed, and were compensated. 


\section{Results and Discussion}

Because people are likely to spend less money when paying with cash (for both practical as well as psychological reasons), we included the transaction medium in the following analyses. Not including the transaction medium, however, does not meaningfully alter the significance levels of any of the following analyses.

A 2 (account size: small or large) $\times 3$ (transaction medium: credit card, debit card, or cash) between-participants ANOVA revealed two significant main effects. Consistent with our first hypothesis, participants who considered large accounts before shopping spent significantly more than did participants who considered small accounts before shopping $\left(F(1,106)=4.30, p=.04, \eta^{2}=.04\right.$; see table 1$)$. Transaction medium also significantly affected spending $\left(F(2,106)=6.48, p=.002, \eta^{2}=.11\right)$. Post hoc contrasts (Fisher's least significant difference) revealed that participants spent less when paying with cash $\left(M_{\text {cash }}=\$ 6.65\right.$, $\mathrm{SD}=5.82)$ than when paying with a credit card $\left(M_{\text {cred. }}=\$ 11.45, \mathrm{SD}=7.69\right)$ or a checking or debit card $\left(M_{\text {ch./deb. }}=\$ 11.08, \mathrm{SD}=6.56\right), p$ 's $<.01$. The latter two transaction mediums did not differ significantly from each other $(p=.87)$. The account size by transaction medium interaction was not statistically significant $(F(1,106)=$ 2.37, $\left.p=.10, \eta^{2}=.04\right)$.

It appears that an accessible account's size influences the consumers' purchase decisions. Independent of any actual differences in wealth or changes in the display or cost of items, customers who thought about large financial accounts before shopping spent more than customers who thought about a smaller financial account before shopping. Indeed, customers who thought about their checking and savings accounts before shopping spent $36 \%$ more than did customers who thought about items in their wallet. The results also replicate previous findings about the importance of transaction medium in consumption, as customers spent more when paying with a credit card than when paying with cash (Feinberg 1986; Hirschman 1979; Prelec and Simester 2001). Of course, as participants were not randomly assigned to the transaction medium in this experiment, participants lacking sufficient cash for larger purchases may have simply elected to use a credit or checking card.

\section{EXPERIMENT 2: CALORIC CONSUMPTION}

Experiment 2 tested whether a manipulation of account accessibility would moderate consumption in a completely different domain of consumption - the consumption of calories. Participants were first informed of a food's (i.e., M\&M's) impact on a small or large account of their caloric resources and were then asked several filler questions. At the end of the experiment, participants were invited to take whatever amount of M\&M's they wanted from a bowl as thanks for their participation. The amount of M\&M's participants removed from the bowl served as the critical dependent measure. We predicted that participants would take fewer M\&M's when small caloric resource accounts were cognitively accessible than when larger caloric resource accounts were cognitively accessible (hypothesis 1 ).

\section{Method}

Participants. Thirty-four pedestrians in Cambridge, Massachusetts (16 males and 18 females, $M_{\text {age }}=19.7$, $\mathrm{SD}=9.1$ ), completed a short survey and were offered M\&M's as compensation.

Procedure. Participants were invited to complete a survey in exchange for M\&M's and were handed a questionnaire that contained all manipulations and measures. Participants in the small account condition first read that a standard package of M\&M's constitutes 3/25 of one's rec-

TABLE 1

OBJECTIVE COST, SUBJECTIVE COST, AND CONSUMPTION BY SIZE OF COGNITIVELY ACCESSIBLE RESOURCE ACCOUNT IN EXPERIMENTS 1-4

\begin{tabular}{|c|c|c|c|c|}
\hline \multirow[b]{2}{*}{ Dependent measure } & \multicolumn{4}{|c|}{ Cognitively accessible account } \\
\hline & Small & SD & Large & SD \\
\hline \multicolumn{5}{|l|}{ Experiment 1: financial consumption: } \\
\hline Amount spent & $\$ 6.68$ & 4.55 & $\$ 9.09$ & 7.83 \\
\hline \multicolumn{5}{|l|}{ Experiment 2: caloric consumption: } \\
\hline M\&M's taken (grams) & 4.72 & 5.11 & 10.31 & 9.53 \\
\hline \multicolumn{5}{|l|}{ Experiment 3: caloric consumption: } \\
\hline Account size (calories) & $2,114.29$ & 621.92 & $16,845.45$ & $17,374.01$ \\
\hline Objective cost (calories) & 307.02 & 144.74 & 307.73 & 149.26 \\
\hline Subjective cost (how fattening) & 2.67 & 1.52 & 2.04 & 1.84 \\
\hline WTP for M\&M's & $\$ .26$ & .28 & $\$ .40$ & .44 \\
\hline \multicolumn{5}{|l|}{ Experiment 4: temporal consumption: } \\
\hline Objective cost (hours) & 1.31 & 1.06 & 2.16 & 3.08 \\
\hline Subjective cost (how painful) & 4.01 & 1.39 & 3.17 & 1.33 \\
\hline WTP for a cleaning service & $\$ 7.05$ & 8.90 & $\$ 3.62$ & 8.65 \\
\hline
\end{tabular}

NOTE. - WTP $=$ willingness to pay. 
ommended daily caloric intake. Those in the large account condition first read that a standard package of M\&M's constitutes 3/175 of one's recommended weekly caloric intake. To disguise the true nature of the experiment, participants then answered two filler questions, one asking them to briefly describe the first time they tasted M\&M's and another asking them to guess the year in which M\&M's were invented (M\&M's were invented in 1940). Finally, each participant was presented with a small bowl containing 40 grams of M\&M's and told, "As thanks for your participation you can take as many M\&M's as you'd like." The remaining contents of this bowl were subsequently placed in a Ziploc bag and (privately) weighed to determine the actual amount of M\&M's consumed by each participant.

\section{Results and Discussion}

In accordance with our first hypothesis, participants in the large account condition removed more M\&M's from the bowl than did participants in the small account condition $\left(F(1,32)=4.69, p=.04, \eta^{2}=.13\right.$; see table 1$)$. In other words, participants who learned how a food would deplete a relatively small account of calories consumed fewer calories than did participants who learned how a food would deplete a relatively larger account of calories.

Together, the results of the first two experiments suggest that accessible accounts can influence consumption (hypothesis 1) whether the account is simply considered (experiment 1) or the cost is deliberately framed in terms of the depletion of a smaller or larger account (experiment 2). Furthermore, as the objective costs of goods and foods in both of these experiments were constant across conditions, the results suggest that accessible accounts may influence consumption independent of an expense's objective cost (consistent with hypothesis 2). The remaining two experiments directly tested our second and third hypothesis by investigating whether the impact of an accessible resource account on consumption is mediated by changes in the subjective costs of the potential expenditure. Both experiments did so by manipulating the accessibility of large versus small resource accounts and then measuring the subjective costs, objective costs, and the consumption of both calories and time.

\section{EXPERIMENT 3: CALORIC ACCOUNTS, COSTS, AND CONSUMPTION}

Experiment 3 measured caloric consumption using a different procedure that tested our second and third hypotheses. Participants were first asked to estimate either their daily or weekly caloric intake. Participants then indicated the subjective and objective caloric costs of a standard package of M\&M's and last indicated how much they would be willing to pay for a small cup of M\&M's as an index of actual consumption. We used willingness to pay (WTP) in this case to connect with consumer purchasing and to employ a measure directly related to the subjective desirability of that commodity's consumption (Ariely, Loewenstein, and Prelec
2003). Consistent with the previous findings, we predicted that the size of the cognitively accessible resource account would influence estimates of the subjective cost of M\&M's (hypothesis 2), would have little influence on estimates of the objective caloric cost of M\&M's (hypothesis 2), and would influence how much participants were willing to pay for M\&M's (hypothesis 1), and we predicted that changes in the subjective cost would mediate the impact of the account size manipulation on the amount that participants would be willing to pay for M\&M's (hypothesis 3).

\section{Method}

Participants. One hundred and sixty-one undergraduates (55 males and 106 females, $M_{\text {age }}=20.5, \mathrm{SD}=1.4$ ) responded to an e-mail and completed a short online questionnaire.

Procedure. Participants completed a short one-page questionnaire containing the key manipulation and all dependent measures. Participants in the small account condition first estimated their average daily caloric intake, whereas participants in the large account condition first estimated their weekly caloric intake. Next, participants estimated the absolute number of calories contained in a standard package of M\&M's. Participants then rated how fattening they considered M\&M's to be on an 11-point Likert scale marked with end points "not at all fattening $(-5)$ " and "very fattening (+5)." Participants also reported the greatest sum of money that they would be willing to pay for a paper Dixie cup filled with M\&M's by choosing a sum between zero and $\$ 3.00$ (ascending in $\$ .25$ increments). The order of the last two questions was counterbalanced.

\section{Results and Discussion}

There were no significant order effects. As expected, a manipulation check revealed that participants in the small account condition reported consuming fewer calories in an average day than participants in the large account condition reported consuming in an average week $(F(1,159)=$ 60.34, $p<.001, \eta^{2}=.28$; for all means, see table 1$)$.

To test our first and second hypotheses, participants' estimates of the objective caloric costs and subjective costs of M\&M's were transformed into $z$-scores, so those estimates (made in different response formats) could be compared directly across conditions. A 2 (account size: daily vs. weekly caloric intake) $\times 2$ (cost: objective vs. subjective) ANOVA with repeated measures on the second factor yielded a marginally significant account size $\times$ cost interaction $\left(F(1,159)=3.45, p<.07, \eta^{2}=.02\right)$. There was no significant main effect of type of cost $(F<1)$ or account size $\left(F(1,159)=2.66, p=.13, \eta^{2}=.01\right)$. Consistent with our second hypothesis, simple effects tests revealed that participants who reported their average daily caloric intake perceived M\&M's to be more fattening than did participants who reported their weekly caloric intake $(F(1,159)=$ $\left.5.62, p=.02, \eta^{2}=.03\right)$, but there was no significant dif- 
ference in the estimated number of total calories contained in a package of M\&M's $(F<1)$. Consistent with our first hypothesis, participants in the daily caloric intake condition also reported a lower willingness to pay for the quantity of M\&M's than did participants in the weekly caloric intake condition $\left(F(1,159)=5.79, p=.02, \eta^{2}=.04\right)$. Including the objective cost of M\&M's (the estimated number of calories a standard package contains) as a covariate in a ANCOVA does not meaningfully alter the main effect of the accessible account's size on subjective cost and WTP $\left(F(1,158)=5.80, p=.02, \eta^{2}=.04\right.$, and $F(1,158)=$ $6.36, p=.01, \eta^{2}=.04$, respectively).

Mediational Analysis. To test our third hypothesis - whether assessments of subjective costs mediated the impact of account size condition on WTP - we followed a mediational procedure described by Baron and Kenny (1986). First, the independent variable (account size condition) significantly predicted the proposed mediator of subjective cost (how fattening participants considered a package of M\&M's to be; $\beta=-.19, t=2.37, p=.02$ ), and the proposed mediator predicted the dependent variable (WTP; $\beta=-.26, t=3.31, p<.001)$. Second, the independent variable (account size condition) significantly predicted the dependent variable (participants' WTP for M\&M's; $\beta=.19$, $t=2.41, p=.02$ ). Finally, when the mediator (subjective cost) was added to the full model, the mediator significantly predicted the dependent variable (WTP; $\beta=-.23, t=$ 2.95, $p=.004$ ), while the independent variable only predicted the outcome variable at a marginally significant level $(B=.14, t=1.88, p=.06)$. This reduction in the significance level of the independent variable when the mediator was included in the model was confirmed by a Sobel (1982) test $(z=1.85, p=.06)$. This pattern of results is consistent with full mediation (see fig. 1).

In short, participants who first considered a small account of their caloric resources thought that the subjective but not objective cost of a package of M\&M's was higher than did participants who first considered a larger account of their caloric resources. Consequently, participants who considered a small account of their caloric resources were willing to pay less for M\&M's than were participants who considered a larger account of their caloric resources.

\section{EXPERIMENT 4: TEMPORAL ACCOUNTS, COSTS, AND CONSUMPTION}

A final experiment expanded on the previous demonstrations in one additional consumption domain-the consumption of time. As with the consumption of money and calories, the amount of available time can be considered in multiple ways. Mowing the lawn, for instance, may deduct 2 hours from one's day, one's weekend, or one's week. Whether one chooses to expend this time or hire a lawn service to save this time may therefore depend on the account of temporal resources cognitively accessible at the time of judgment. Although consumer researchers have noted important differences between the consumption of
FIGURE 1

MEDIATIONAL ANALYSIS FOR EXPERIMENT 3

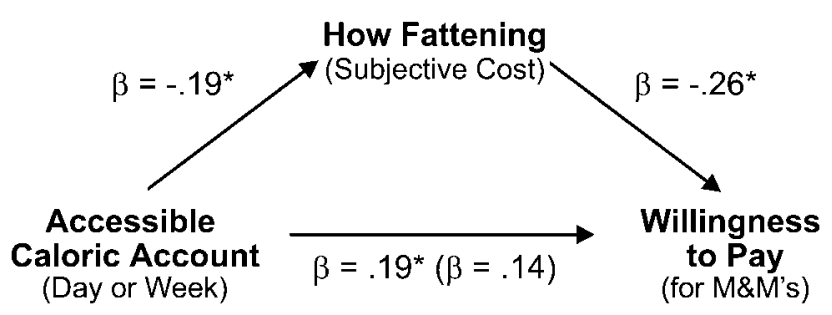

NotE. - Values in parentheses indicate the effect of the accessible caloric account on willingness to pay when the mediator is included in the model; * indicates significant relationships $(p<.05)$.

time and money, whether a particular consumption of time is considered affordable or not should depend on whatever time one appears to have available for consumption (e.g., Leclerc et al. 1995; Okada and Hoch 2004; Soman 2001), just as financial and caloric expenses were considered affordable or not according to the resources that appeared available for consumption in experiments 1-3.

Experiment 4 again tested all three of our hypotheses simultaneously by asking university students to consider the time required to clean their dorm rooms. Most important, we predicted (consistent with hypotheses 2 and 3 ) that participants who considered a small temporal account (time in a day) would be less interested in spending time cleaning their room than those who considered a large temporal account (time in a week), because that cleaning would appear subjectively more painful. Participants reported their interest in spending time cleaning by indicating how much they would be willing to pay for a cleaning service. We again predicted a significant interaction: participants who were less interested in spending time cleaning their room should be more willing to spend money for a cleaning service. As a result, we predicted (consistent with hypothesis 1) that participants who considered a small account should be willing to pay more than participants who considered a larger account. We also predicted (consistent with hypothesis 2 ) that there would be a significantly smaller effect of accessible accounts' sizes on participants' ratings of the absolute amount of time they spent cleaning their rooms.

\section{Method}

Participants. One hundred and twenty-two undergraduate students (57 males and 65 females, $M_{\text {age }}=20.7$, $\mathrm{SD}=1.5)$ volunteered to complete an online survey. One participant's WTP was more then 10 standard deviations from the overall mean and was therefore excluded from the following analyses.

Procedure. Participants in the small account condition were asked to estimate the total amount of time spent each time they cleaned their dorm room on a day that they cleaned, whereas participants in the large account condition 
estimated the amount of time spent each time they cleaned their dorm room in a week that they cleaned. Next, participants reported how annoying and inconvenient cleaning their room was and much time each individual cleaning felt like it took on seven-point scales with end points "not at all/no time at all (1)" and "very annoying/very inconvenient/ a lot of time (7)." Finally, participants indicated how much they would be willing to pay (WTP) a cleaning service to clean their room once.

\section{Results and Discussion}

Participants' responses on the subjective cost assessments of how annoying and inconvenient cleaning their room was and of how long they felt that cleaning it took were highly intercorrelated $(\alpha=.83)$ and were therefore averaged into a composite of subjective cost to simplify analyses. Next, participants' estimates of time spent, reports of subjective cost, and WTP were transformed to $z$-scores so that they could be compared directly and used to test our first two hypotheses.

A 2 (account size: day vs. week) $\times 2$ (cost: objective time spent vs. subjective cost) mixed-model ANOVA with repeated measures on the second factor revealed a significant account size $\times$ cost interaction $(F(1,119)=16.21, p<$ $.001, \eta^{2}=.12$ ) and no main effects (both $F_{\mathrm{S}}<1$ ). As one can see in table 1 , participants in the small account condition reported that a cleaning actually incurred a lower objective cost of their time than did participants in the large account condition $\left(F(1,119)=4.85, p=.03, \eta^{2}=.04\right)$. This appears somewhat inconsistent with the findings of experiment 3 , but note that participants were not explicitly asked to think of the same cleaning episode (as they were with the caloric content of M\&M's), and it is likely that the amount of cleaning done in a week simply contains more episodes than the cleaning done in a day. More important for our main predictions, however, participants in the small account condition also reported that the subjective cost of cleaning a single time was significantly larger than did participants in the large account condition (consistent with hypothesis $2 ; F(1,119)=10.95, p=.001, \eta^{2}=.08$; for all means, see table 1$)$.

A 2 (account size: day vs. week) $\times 2$ (consumption: objective time spent vs. WTP) mixed-model ANOVA with repeated measures on the second factor yielded only the predicted significant interaction $(F(1,119)=10.44, p=$ $\left..002, \eta^{2}=.08\right)$. Again, although participants in the small account condition reported spending less objective time cleaning than did participants in the large account condition, participants in the small account condition were willing to pay more to have a service perform a one-time cleaning (consistent with hypothesis $1 ; F(1,119)=4.32, p=.04$, $\left.\eta^{2}=.04\right)$. Including time spent as a covariate in an ANCOVA does not meaningfully alter the effect of account size on subjective cost or WTP $(F(1,118)=11.46, p=$ $.001, \eta^{2}=.09$, and $F(1,118)=5.34, p=.02, \eta^{2}=.04$, respectively).
Mediational Analysis. To test our third hypothesis, we again performed a mediational analysis by following the three-step procedure described by Baron and Kenny (1986). This analysis is shown in figure 2. First, the independent variable (account size condition) significantly predicted the proposed mediator of subjective cost $(\beta=-.29, t=3.31$, $p=.001)$, and the proposed mediator predicted the dependent variable (WTP; $\beta=.36, t=4.23, p<.001$ ). Second, the independent variable (account size) also significantly predicted the dependent variable (WTP; $\beta=-.19, t=2.08$, $p=.04$ ). Finally, when the mediator (subjective cost) was added to the full model, the mediator significantly predicted the dependent variable $(\beta=.36, t=3.76, p<.001)$, while the independent variable was no longer a significant predictor of the dependent variable $(\beta=-.09, t=1.00, p=.32)$. This reduction in the significance level of the independent variable when the mediator was included in the model was significant by a Sobel (1982) test $(z=2.49, p=.01)$.

Although participants who considered a small temporal account reported spending less objective time cleaning their room than participants who considered a larger temporal account, they reported that cleaning was more painful. Consequently, these participants were willing to pay more money for a cleaning service to save them from consuming objectively less time. These results again highlight the importance of accessible resources as a determinant of consumption and again demonstrate the different effects such manipulations have on objective versus subjective evaluations of the costs associated with consumption. In experiment 4 , subjective costs influenced consumption not just in the absence of changes in objective costs but, rather, in spite of them.

\section{GENERAL DISCUSSION}

Decisions to consume a commodity depend both on the cost of consumption ("How much does it cost?") as well as the resources available for consumption ("How much can I afford to consume?"). Identifying the objective cost of consumption is relatively easy, but identifying the resources available for consumption is often considerably more dif-

\section{FIGURE 2}

\section{MEDIATIONAL ANALYSIS FOR EXPERIMENT 4}

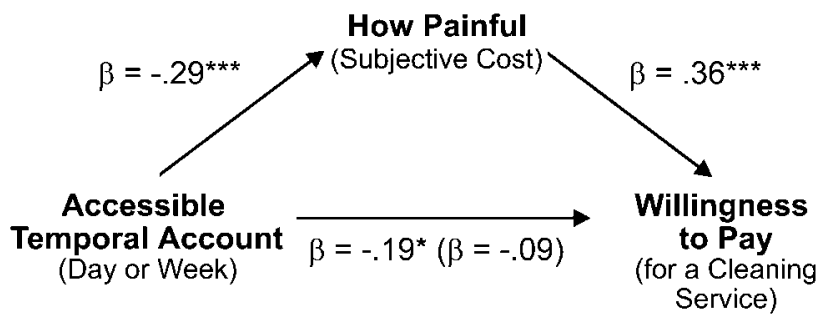

NOTE. - Values in parentheses indicate the effect of the accessible temporal

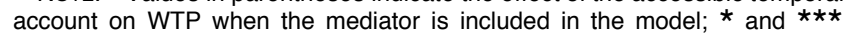
indicate significant relationships $(p<.05$ and $p<.001$, respectively). 
ficult. Instead of attempting to calculate the total amount of resources available for consumption when making decisions, consumers appear to assess the potential impact of a decision on the resource account that is cognitively accessible. Consumers employ different budgets and transaction mediums to regulate their consumption of different kinds of expenses (Heath and Soll 1996; Prelec and Simester 2001; Thaler 1985). The present research suggests that not only are categories of mental accounts and transaction mediums influential but that, irrespective of one's total wealth, the very amount of resources in a specific account influences how freely resources are spent. For example, travelers may spend money more liberally while on vacation than when at home because they carry more cash than is their usual custom.

Whether considering money, calories, or time, participants in our experiments considered a commodity's subjective cost to be less and, consequently, consumed more of a commodity when large resource accounts were made cognitively accessible than when small resource accounts were made cognitively accessible. The consistency of these results across different domains of consumption, as well as across different manipulations of resource accessibility, suggests that these findings are likely to be a general feature of consumption rather than an idiosyncratic feature of any single experiment. Thus, contextual information influences a good's apparent value by altering the apparent size of the numerator in our simple consumption equation (i.e., the absolute cost of a good) but may also influence a good's apparent value by determining which denominator (i.e., resource account) is considered. Indeed, just as commodities appear more valuable when compared with more expensive goods (Gourville 1998; Herr 1989), they also appear more affordable when relatively large resource accounts just happen to be accessible because their subjective costs appear smaller in comparison.

The current experiments demonstrate that manipulating the cognitive accessibility of financial, caloric, and temporal resource accounts influences the subjective costs and consumption of those resources, but they do not address when consumers are naturally likely to think about their resources in terms of small versus large accounts. We think that there are likely to be at least four important determinants of these natural assessments that future research could elucidate. First, and most important, we suspect that the method of payment may naturally activate different resource accounts in financial consumption. As mentioned, Prelec and Simester (2001) found that participants in an auction bid more money when paying with a credit card than when paying with cash (Feinberg 1986; Hirschman 1979). Prelec and Simester offered no explicit theory to explain this difference but briefly suggested that credit cards may lead people to "anchor" on their relatively high credit limit and therefore spend more money. Instead, the present research implies that the mere consideration of a large resource account-such as one's available credit-can increase consumption and that this is at least a plausible mechanism for differences in consumption between payment methods. Those methods that natu- rally activate thoughts about relatively large resource accounts, such as credit cards, are more likely to decrease the subjective cost of an item and increase the likelihood of consumption. Those methods that naturally activate thoughts about relatively small resource accounts, such as cash payments, are more likely to increase the subjective cost of an item and decrease the likelihood of consumption.

Second, just as a daily expense leads consumers to compare it to other daily expenses such as the cost of a newspaper (Gourville 1998), we suspect that the objective cost of an item may lead people to consider resource accounts of similar size. People are unlikely to think spontaneously of their total net worth when buying a candy bar but are considerably more likely to do so when buying a new car. This predicts that people would report being subjectively less wealthy after considering low-cost items than after considering high-cost items, even though their ability to actually purchase the items considered would be dramatically higher in the former case than in the latter.

Third, we suspect that one's temporal perspective may influence the natural accessibility of small versus large resource accounts. Research suggests that people tend to construe near-future events at a lower conceptual level than distant-future events (Chandran and Menon 2004; Trope and Liberman 2000), thinking about near-future events in terms of their concrete details and feasibility and thinking about distant future events in terms of their more abstract features and overall desirability. This may therefore lead people considering a near-future expense to consider smaller resource accounts (such as the money in their wallets or time available in a day), to feel subjectively less wealthy, and to consume fewer resources than people considering a distant-future expense. In addition, if these temporal frames lead people to consider different resource accounts, then one's temporal perspective should influence not only consumption of near and distant-future expenses but also one's current consumption. If people feel wealthier after considering distantfuture expenses, then they may be more inclined to consume a commodity right now than people who have considered near-future expenses.

Finally, the desirability of an item may influence whether a large or small resource account is cognitively accessible when consumers determine if that expenditure is justifiable. When a desirable expense (e.g., dinner at a restaurant) can be assigned to multiple categories of mental accounts (e.g., food or entertainment), consumers justify that expenditure by considering it to influence an applicable mental account containing a surplus rather than a deficit of resources (Cheema and Soman 2006). Consumers may similarly first consider large resource accounts when determining whether desirable expenses can be justified and first consider small resource accounts when determining whether undesirable expenses can be justified. When determining whether they have time to watch a movie, for example, people may naturally consider that activity's impact on the amount of free time they have that week. When determining whether they have time to exercise, however, people may naturally con- 
sider that activity's impact on the amount of free time they have that day. In other words, processes involved in motivated reasoning (Pyszczynski and Greenberg 1987) may influence which resource account is cognitively accessible at the time of a consumption decision.

Knowing the resources available for consumption is an obvious requirement for any decision maker, as consumption depends on not only the cost of a commodity but also whether one has the resources to afford its consumption. Traditional economic theory assumes such calculations are not only possible but routinely computed by consumers. The present research casts doubt on this assumption by demonstrating that simply activating large versus small resource accounts can influence people's consumption of a wide variety of commodities. Whether the financial, temporal, or caloric price of a commodity seems worth consuming appears to depend critically on how that expense would deplete the resource account cognitively accessible at the time of consumption.

\section{REFERENCES}

Ando, Albert and Franco Modigliani (1963), "The 'Life Cycle' Hypothesis of Saving: Aggregate Implications and Tests," American Economic Review, 53 (March), 55-84.

Ariely, Dan, George Loewenstein, and Drazen Prelec (2003), "Coherent Arbitrariness: Stable Demand Curves without Stable Preferences," Quarterly Journal of Economics, 118 (1), 73-105.

Baron, Reuben M. and David A. Kenny (1986), "The ModeratorMediator Variable Distinction in Social Psychological Research: Conceptual, Strategic, and Statistical Considerations," Journal of Personality and Social Psychology, 51 (6), 1173-82.

Chandran, Sucharita and Geeta Menon (2004), "When a Day Means More than a Year: Effects of Temporal Framing on Judgments of Health Risk," Journal of Consumer Research, 31 (September), 375-89.

Cheema, Amar and Dilip Soman (2006), "Malleable Mental Accounting: The Effect of Flexibility on the Justification of Attractive Spending and Consumption Decisions," Journal of Consumer Psychology, 16 (1), 33-44.

Feinberg, Richard A. (1986), "Credit Cards as Spending Facilitating Stimuli: A Conditioning Interpretation," Journal of Consumer Research, 13 (December), 348-56.

Gourville, John T. (1998), "Pennies-a-Day: The Effect of Temporal Reframing on Transaction Evaluation," Journal of Consumer Research, 24 (March), 395-408.

Gourville, John T. and Dilip Soman (1998), "Payment Depreciation: The Behavioral Effects of Temporally Separating Pay- ments from Consumption," Journal of Consumer Research, 25 (September), 160-74.

Heath, Chip and Jack B. Soll (1996), "Mental Budgeting and Consumer Decisions," Journal of Consumer Research, 23 (June), $40-52$.

Helson, Harry (1964), Adaptation-Level Theory, New York: Harper \& Row.

Herr, Paul (1989), "Priming Price: Prior Knowledge and Context Effects," Journal of Consumer Research, 16 (June), 67-75.

Hirschman, Elizabeth C. (1979), "Differences in Consumer Purchase Behavior by Credit Card Payment System," Journal of Consumer Research, 6 (June), 58-66.

Hsee, Christopher K., George F. Loewenstein, Sally Blount, and Max H. Bazerman (1999), "Preference Reversals between Joint and Separate Evaluation of Options: A Review and Theoretical Analysis," Psychological Bulletin, 125 (5), 576-90.

Kahneman, Daniel and Dale T. Miller (1986), "Norm Theory: Comparing Reality to Its Alternatives," Psychological Review, 93 (2), 136-53.

Kahneman, Daniel and Amos Tversky (1979), "Prospect Theory: An Analysis of Decision under Risk," Econometrica, 47 (March), 263-91.

Leclerc, France, Bernd H. Schmitt, and Laurette Dubé (1995), "Waiting Time and Decision Making: Is Time like Money?" Journal of Consumer Research, 22 (June), 110-19.

Okada, E. M. and S. J. Hoch (2004), "Spending Time versus Spending Money," Journal of Consumer Research, 31, 313-23.

Parducci, Allen (1995), Happiness, Pleasure, and Judgment: The Contextual Theory and Its Applications, Mahwah, NJ: Erlbaum.

Prelec, Drazen and George Loewenstein (1998), "The Red and the Black: The Mental Accounting of Savings and Debt," Marketing Science, 17 (1), 4-28.

Prelec, Drazen and Duncan Simester (2001), "Always Leave Home without It: A Further Investigation of the Credit-Card Effect on Willingness to Pay," Marketing Letters, 12 (1), 5-12.

Pyszczynski, Thomas and Jeff Greenberg (1987), "Toward an Integration of Cognitive and Motivational Perspectives on Social Inference: A Biased Hypothesis-Testing Model," in Advances in Experimental Social Psychology, Vol. 20, ed. Leonard Berkowitz, San Diego, CA: Academic Press, 297-340.

Sobel, Michael E. (1982), "Asymptotic Confidence Intervals for Indirect Effects in Structural Equation Models," Sociological Methodology, 13, 290-312.

Soman, Dilip (2001), "Effects of Payment Mechanism on Spending Effects: The Role of Rehearsal and Immediacy of Payments," Journal of Consumer Research, 27 (March), 460-74.

Soman, Dilip and Amar Cheema (2002), "The Effects of Credit on Spending Decisions: The Role of Credit Limit and Credibility," Marketing Science, 21 (1), 32-53.

Thaler, Richard H. (1985), "Mental Accounting and Consumer Choice," Marketing Science, 4 (Summer), 199-214.

Trope, Yaacov and Nira Liberman (2000), "Temporal Construal and Time-Dependent Changes in Preference," Journal of Personality and Social Psychology, 79 (December), 876-89. 